\title{
Utilisation des spermatozoïdes testiculaires en ICSI. Intérêt de la culture in vitro. Revue de la littérature
}

\author{
B. SCHUBERT, G. GRIZARD, D. BOUCHER \\ Laboratoire de Biologie du Développement et de la Reproduction-CECOS, Hôtel-Dieu, Clermont-Ferrand
}

\section{RÉSUMÉ}

Au cours de ces dernières années les tentatives d'ICSI réalisées avec des spermatozoïdes testiculaires se sont considérablement accrues. Cependant, l'une des difficultés techniques tient au fait que la mobilité des spermatozoïdes est extrêmement réduite. Un accroissement de la mobilité avait été observé après une incubation des prélèvements testiculaires pendant quelques heures. Dans l'optique d'augmenter les chances de succès, une culture in vitro des spermatozoïdes, préalable à l'ICSI, a été envisagée. Nous rapportons ici la revue de la littérature sur ce sujet.

Dans les azoospermies obstructives, la culture in vitro présente peu d'intérêt puisque, excepté une éventuelle "maturation" des spermatozoïdes pendant cette période, un pourcentage appréciable de spermatozoïdes mobiles est souvent observé rapidement après le traitement de la biopsie.

Dans les azoospermies non obstructives la culture in vitro paraît justifiée, puisque lorsque l'on retrouve des spermatozoïdes dans la biopsie, ils sont généralement immobiles. Cependant, la plupart des auteurs s'accordent à reconnaître que les résultats restent aléatoires : au terme de la culture, soit il existe un gain de mobilité, soit au contraire la plupart des spermatozoïdes sont morts.

Quelque soit le type d'azoospermie, les meilleurs résultats sont obtenus après 3-4 jours de culture. La supplémentation des milieux de culture avec de la FSH recombinante s'avère aussi efficace.

La culture in vitro peut être associée à la congélation des spermatozoïdes et la séquence culture in vitro/congélation donnerait de meilleurs résultats que la séquence congélation/culture in vitro.

Les quelques travaux sur l'utilisation en ICSI des spermatozoïdes maintenus en culture in vitro pendant 1 à 2 jours rapportent des résultats satisfaisants en terme de taux de fécondation et de grossesse.

La culture in vitro de spermatozoïdes testiculaires peut constituer une voie de recherche intéressante pour améliorer les résultats en ICSI, dans les cas où la quantité de spermatozoïdes retrouvée et/ou leur mobilité sont très faibles. De plus, elle nous paraît être un bon modèle d'étude des mécanismes d'acquisition de la mobilité des spermatozoïdes.

Mots clés : spermatozoïdes testiculaires, culture in vitro, azoospermie, congélation, décongélation.

Correspondance : B. Schubert, Laboratoire de Biologie du Développement et de la Reproduction - CECOS, HôtelDieu, Boulevard Léon Malfreyt, 63000 Clermont-Ferrand e-mail : bschubert@chu-clermontferrand.fr

Prix de DESS décerné par la Société d'Andrologie de Langue Française. Présentation au XVIlème Congrès de la SALF, 7-8 décembre 2000, Bordeaux. 


\section{INTRODUCTION}

L'avènement de l'injection intracytoplasmique de spermatozoïdes (ICSI) a permis à des hommes réputés jusqu'alors stériles de procréer [31]. Plus récemment, en utilisant en ICSI des spermatozoïdes testiculaires, des enfants sont nés au sein de couples dont l'homme présentait une azoospermie. Le pronostic de ces hommes en terme de fertilité a donc radicalement changé. L'exploration des azoospermies permet classiquement de les classer en 2 grands groupes : les azoospermies obstructives et les azoospermies non obstructives. Dans ces deux grandes classes d'azoospermie, même si les étiologies sont très différentes, des naissances ont été rapportées [7, 37, 38]. Cependant, dans 25 à $30 \%$ des azoospermies, la biopsie testiculaire ne retrouve pas de spermatozoïdes, sans qu'aucun facteur clinique ni biologique ne puisse le prédire [23]. Ce chiffre s'élève jusqu'à $40 \%$ dans les azoospermies non obstructives [32].

Aussi la cryoconservation de ces prélèvements s'est développée. La réalisation préalable de la biopsie testiculaire permet d'éviter une stimulation et une ponction ovarienne inutile si aucun spermatozoïde n'était retrouvé.

Les spermatozoïdes testiculaires frais donnent en général des taux de fécondation plus faibles que les spermatozoïdes éjaculés frais : 47,6\% versus 58,7 \% [11]. Les manipulations préalables pourraient aussi intervenir puisque avec des spermatozoïdes congelés/décongelés, les résultats en ICSI seraient plus faibles qu'avec des spermatozoïdes testiculaires frais [6], ce qui n'est cependant pas vérifié par d'autres auteurs $[12,14]$ qui retrouvent des résultats comparables dans les 2 situations. De même, concernant la nature de l'azoospermie, les résultats sont contradictoires [12, 33, 39].

Toutes ces données sont en réalité difficilement comparables entre elles. La nature de l'azoospermie n'est pas toujours précisée et la comparaison des résultats obtenus avec des spermatozoides frais et des spermatozoïdes congelés/décongelés n'est pas toujours aisée. Bien que l'utilisation de spermatozoïdes immobiles puisse permettre l'obtention de grossesses [22, 27, 34, 36], les spermatozoïdes testicu- laires donnant alors de meilleurs résultats que les spermatozoïdes éjaculés et immobiles [16, $22,36]$, il est un point sur lequel les auteurs s'accordent : l'utilisation de spermatozoïdes mobiles donne toujours les meilleurs résultats en ICSI [17, 22, 27, 24, 26, 35].

Classiquement la mobilité des spermatozoïdes apparaît au cours de la traversée de l'épididyme [22]. Dans les prélèvements testiculaires, des spermatozoïdes mobiles peuvent cependant être retrouvés après dilacération des tissus et incubation quelques heures à $37^{\circ} \mathrm{C}$ dans des milieux de cultures classiques. Ces observations ont été publiées initialement par Nagy et al. [25] : quelques minutes après la biopsie testiculaire, tous les spermatozoïdes étaient immobiles puis après une demi-heure à 2 heures d'incubation, plusieurs spermatozoïdes se sont révélés mobiles (non progressifs). Craft et al. [5] ont remarqué qu'en laissant les prélèvements testiculaires à $37^{\circ} \mathrm{C}$ pendant 24 à 72 heures, il existe une augmentation significative du nombre de spermatozoïdes mobiles progressifs, et particulièrement entre 48 et 72 heures. D'ailleurs ces auteurs peinent à croire qu'une telle proportion de spermatozoïdes puissent "come to life". Ils n'apportent pas d'explication à ces résultats mais envisagent la possibilité de facteurs bloquant la mobilité dans le testicule et qui auraient une action diminuée après leur dilution dans les milieux d'incubation. Les spermatozoïdes testiculaires, reconnus comme immobiles, pourraient alors acquérir une mobilité après une culture in vitro plus ou moins prolongée. Toutes ces observations peuvent servir de base pour faire évoluer les techniques d'ICSI.

Nous rapportons ici les données de la littérature concernant l'effet de la "culture in vitro" sur la qualité des spermatozoïdes testiculaires et les conséquences éventuelles sur les résultats en ICSI.

Notons que le terme de "culture in vitro" utilisé par les auteurs anglo-saxons est un abus de langage. Il correspond en réalité à une technique in vitro de maintien en survie des spermatozoïdes.

Nous n'aborderons pas les cas d'immobilité majeure des spermatozoïdes éjaculés liés à des 
pathologies flagellaires telles que l'absence de bras de dynéine, le syndrome des flagelles courts, pour lesquelles bien évidemment aucune acquisition de mobilité n'est à attendre dans ces anomalies structurales. Il reste cependant possible que, dans les cas que nous allons évoquer, des azoospermies soient associées à de telles pathologies, mais elles sont probablement très rares.

De même nous n'aborderons pas le cas des spermatozoïdes épididymaires. En effet, dans les ponctions épididymaires réalisées dans les azoospermies obstructives, la mobilité est déjà appréciable et la culture in vitro n'entraîne aucune amélioration $[8,29]$.

\section{TECHNIQUES ET ANALYSES GENERALEMENT UTILISEES}

\section{Critères d'appréciation de la mobilité.}

L'évaluation de la mobilité fait le plus souvent référence à la classification de l'OMS [43], en distinguant 2 grands groupes : les spermatozoïdes ayant une mobilité progressive (classification "a" et "b" de l'OMS) et ceux ayant une mobilité réduite ("c"). Cette dernière catégorie fait l'objet de différentes appellations selon les auteurs : mouvements faibles, secousses, tremblements, pour insister sur la recherche très minutieuse d'un mouvement. L'un d'entre eux est encore plus clair en expliquant que chaque spermatozoïde est observé plusieurs secondes,

Tableau 1 : Composition des différents milieux et conditions de culture des spermatozö̈des testiculaires.

\begin{tabular}{|c|c|c|c|c|}
\hline & Milieu de base & $\begin{array}{l}\text { supplément } \\
\text { protéique }\end{array}$ & Temp. & Atmosphère \\
\hline Edirisinghe et al, 1996 [8] & $\begin{array}{l}\text { T6 tamponné à } \\
\text { l'HEPES } \\
\text { HTF tamponné au } \\
\text { bicarbonate }\end{array}$ & $10 \%$ sérum humain & $37^{\circ} \mathrm{C}$ & $\mathrm{air}+5 \% \mathrm{CO}_{2}$ \\
\hline Zhu et al, $1996 ; 1997[44,45]$ & IVF & $?$ & $37^{\circ} \mathrm{C}$ & $\mathrm{air}+5 \% \mathrm{CO}_{2}$ \\
\hline Liu et al, 1997 [19] & $\mathrm{B} 2$ & $3 \%$ SSS & $37^{\circ} \mathrm{C}$ & air $+5 \% \mathrm{CO}_{2}$ \\
\hline Urman et al, 1998 [40] & $\begin{array}{l}\text { EBSS + pénicilline } \\
+ \text { pyruvate }\end{array}$ & HSA & $37^{\circ} \mathrm{C}$ & $?$ \\
\hline $\begin{array}{l}\text { Van den Bergh et al, } 1998[41] \\
\text { Emiliani et al, 1999; } 2000[9,10]\end{array}$ & $\begin{array}{l}\text { EBSS tamponné au } \\
\text { bicarbonate }\end{array}$ & $20 \%$ sérum humain & $\begin{array}{l}32^{\circ} \mathrm{C} \\
37^{\circ} \mathrm{C}\end{array}$ & $\begin{array}{l}90 \% \mathrm{~N} 2+5 \% \\
\mathrm{CO}_{2}+5 \% \mathrm{O}_{2}\end{array}$ \\
\hline Balaban et al, 1999 [3] & $\begin{array}{l}\text { EBSS +/- FSH } \\
\text { recombinante }\end{array}$ & $0,5 \%$ HSA & $30^{\circ} \mathrm{C}$ & $?$ \\
\hline Jaroudi et al, 1999 [15] & IVF & $?$ & ambiante & ambiante \\
\hline Hu et al, 1999 [13] & $\mathrm{HTF}$ & $10 \% \mathrm{SSS}$ & $37^{\circ} \mathrm{C}$ & air $+5 \% \mathrm{CO}_{2}$ \\
\hline Angelopoulos et al, 1999 [2] & $\mathrm{HTF}$ & $6 \%$ Plasmanate & $37^{\circ} \mathrm{C}$ & $\mathrm{air}+5 \% \mathrm{CO}_{2}$ \\
\hline Liu et al, $1999 ; 2000[20,21]$ & $\begin{array}{l}\text { HTF tamponné à } \\
\text { l'HEPES }\end{array}$ & $3 \%$ SSS & $37^{\circ} \mathrm{C}$ & $\mathrm{air}+5 \% \mathrm{CO}_{2}$ \\
\hline
\end{tabular}

Temp. : température d'incubation ; EBSS : Earle's Balanced Salt Solution (Sigma, Aldrich);

FSH recombinante (Puregon, Organon) ; HTF : Human Tubal Fluid (Irvine Scientific) ;

HSA : Human Serum Albumin (Irvine Scientific) ; IVF medium (Medi-Cult, Denmark) ;

Plasmanate (National Hospital Specialties, Hackensack, NJ, USA)

SSS : Substitute Synthetic Serum (Irvine Scientific) ; T6 : ; milieu de type EBSS 
pour permettre, éventuellement, de mettre en évidence de faibles mouvements qui n'auraient pas été observés dans un premier temps [2].

\section{Conditions expérimentales (Tableau 1).}

Les fragments testiculaires dilacérés sont en général déposés, après centrifugation et élimination des plus gros débris cellulaires, dans un milieu de culture en goutte sous huile minérale, puis incubés à $37^{\circ} \mathrm{C}$ sous atmosphère contrôlée à $5 \%$ de $\mathrm{CO}_{2}$ et $95 \%$ d'air. Des températures d'incubation inférieures ont été utilisées : température ambiante, $30^{\circ} \mathrm{C}, 32^{\circ} \mathrm{C}$, mais avec des avantages qui restent limités. A $32^{\circ} \mathrm{C}$ la mobilité se maintient plus longtemps qu'à $37^{\circ} \mathrm{C}$, mais la proportion maximale de spermatozoïdes mobiles obtenue entre le $3 \mathrm{è} \mathrm{et}$ le 4 è jour de culture, est comparable pour les deux températures [41]. Le plus souvent, la culture in vitro n'excède pas 4 jours et aucun changement de milieu n'est effectué pendant cette période.

Les incubations sont généralement réalisées dans des milieux chimiquement définis (Type Earle tamponné au bicarbonate ou à l'Hepes). Ces milieux peuvent être enrichis : ajout d'extraits biologiques, d'hormones... Un apport protéique est réalisé soit par du sérum ou de l'albumine (HSA) à des concentrations variables de $0,5 \%$ à $20 \%$, soit par le Plasmanate ou un supplément appelé " Substitute Synthetic Serum " qui contient en réalité $84 \%$ d'albumine humaine.

Aucun de ces milieux n'a fait la preuve de sa supériorité par rapport aux autres. Seule la FSH recombinante à la dose de $25 \mathrm{mUI} / \mathrm{ml}$ permet une augmentation significative du nombre de spermatozoïdes mobiles après 24 heures de culture [3]. Le mécanisme d'action de la FSH est probablement indirect, puisque les spermatozoïdes ne possèdent pas de récepteurs à cette hormone. La FSH pourrait agir via les cellules de Sertoli, présentes dans les milieux de culture qui exerceraient leur action par contact direct ou par l'intermédiaire de leurs produits de sécrétion selon que la préparation des tissus testiculaires provoque ou non une dispersion des cellules de Sertoli et des spermatozoïdes.

Des supplémentations avec la pentoxifylline et la 2-déoxyadénosine ont aussi été réalisées [2].
Ces molécules permettent d'obtenir en 30 minutes d'incubation une mobilité comparable à celle obtenue après $48 \mathrm{~h}$ de culture in vitro dans des milieux dépourvus de ces substances. Toutefois les risques toxiques potentiels de ces molécules pour l'ovocyte ou l'embryon ne peuvent pas être exclus, même si après la culture, un lavage est toujours réalisé.

En fait, les mécanismes moléculaires mis en jeu dans l'acquisition de la mobilité des spermatozoïdes restent mal connus. Il semblerait cependant que les deux principaux seconds messagers les plus probablement impliqués dans ce processus soient l'AMP $\mathrm{c}_{\mathrm{c}}$ et le $\mathrm{pH}$ [1].

\section{RESULTATS}

\section{Culture in vitro de spermatozoïdes tes- ticulaires frais obtenus dans des azoospermies non étiquetées et dans des azoospermies obstructives (Tableau 2).}

La distinction entre les azoospermies obstructives et les azoospermies non obstructives n'est pas toujours établie. Aussi, dans notre étude nous avons regroupé les résultats se rapportant aux azoospermies non étiquetées et aux azoospermies obstructives, et analysé de façon séparé les résultats relatifs aux azoospermies non obstructives.

Bien que l'expression des résultats diffère selon les équipes, l'ensemble des données montre qu'il existe généralement une amélioration de la mobilité au cours du temps. Au moment du prélèvement testiculaire (J0), la mobilité progressive est soit très faible soit absente et elle augmente progressivement pour atteindre un maximum à $\mathrm{J} 3$ ou $\mathrm{J} 5[8,19,45]$. La mobilité totale, souvent appréciable au moment de la ponction testiculaire, suit approximativement la même évolution au cours de la culture $[2,9,10,13,19,28,29]$. Son maintien dépend des conditions expérimentales, en particulier de la température, puisqu'à $32^{\circ} \mathrm{C}, 20 \%$ de spermatozoïdes mobiles sont encore présents après 10 jours de culture [41], alors qu'à $37^{\circ} \mathrm{C}$ la plupart des spermatozoïdes sont déjà morts après 7-8 jours de culture [19].

Au cours de la culture, il existerait aussi une modification de la morphologie de certains spermatozoïdes avec notamment une perte de 
Tableau 2 : Culture in vitro de spermatozoïdes testiculaires obtenus à partir d'échantillons prélevés dans des azoospermies obstructives ou non étiquetées.

Proportion de patients avec des spermatozoïdes mobiles

$\begin{array}{lllllllll}\text { J0 } & \text { J1 } & \text { J2 } & \text { J3 } & \text { J4 } & \text { J5 } & \text { J6 } & \text { J9 } & \text { J10 }\end{array}$

Zhu et al, 1996* $(n=3)$ [44]

$3 / 3$

Zhu et al, $1997(n=20)$ [45] 18/20

$19 / 20$

$19 / 20$

Urman et al, $1998(\mathbf{n}=10)$ [40] 10/10

$10 / 10$

Hu et al, 1999* $(n=20)[13] \quad 14 / 20$

$18 / 20$

Pourcentage moyen de spermatozoïdes mobiles

Edirisinghe et al, $1996(\mathrm{n}=1) \quad 0$

10

15

25

35

38

282

[8]

Liu et al, $1997(\mathrm{n}=17)$ [19] $\quad \mathbf{2}+62$

$19+50$

$\mathbf{3 3}+37$

$\mathbf{5 3}+19$

$48+31$

$31+23$

Nijs et al, 1997* $(n=35)$ [28]

51

91

Nijs et al, 1998* $(n=5)$ [29]

Van den Bergh et al, 1998*

$(\mathrm{n}=7)$ [41]

53

Angelopoulos et al, 1999

$(\mathrm{n}=7)(\mathrm{a})[2]$

$$
5
$$

9

11

10

4

Angelopoulos et al, 1999

$(\mathrm{n}=3)$ [2]

0

3

4

2

1

Emiliani et al, 1999

$(\mathrm{n}=6)$ [9]

21

54

Emiliani et al, 2000

$(\mathrm{n}=6)$ [10]

17

28

48

47

32

31

$33 \quad 30$

18

J0 à Jn : nombre de jours de culture

Les caractères en gras indiquent la mobilité progressive

* : azoospermies non étiquetées

(a) : seuls sont pris en compte les échantillons présentant des spermatozoïdes mobiles lors de la ponction

restes cytoplasmiques [44], conduisant à un accroissement des spermatozoïdes morphologiquement normaux (3\% versus $21 \%$ ) [19].

\section{Culture in vitro de spermatozoïdes tes- ticulaires frais obtenus dans des azoospermies non obstructives (Tableau 3 , Figure1).}

Les échantillons peuvent être divisés en deux groupes selon la présence ou non de spermatozoïdes mobiles le jour de la ponction.

Lorsqu'une faible mobilité existe, toujours non progressive, celle-ci se maintient ou est améliorée au cours de la culture. Le pourcent- age moyen de spermatozoïdes mobiles augmente et dans certains cas on observe le développement d'une mobilité progressive. Le maximum se situe après $2-4$ jours de culture $[2,45]$. La présence de FSH recombinante dans le milieu de culture aurait un effet bénéfique sur le développement de la mobilité [3].

Si aucun spermatozoïde mobile n'est observé le jour de la ponction testiculaire, pour certaines équipes, la culture n'apporte aucune amélioration $[2,19]$. Il existe même un effet délétère de l'incubation puisque des spermatozoïdes initialement immobiles mais vivants, ne montrent plus aucun signe de vitalité après 24 à 96 heures de culture comme en attestent les col- 
Tableau 3 : Culture in vitro de spermatozoïdes testiculaires obtenus à partir d'échantillons prélevés dans des azoospermies non obstructives.

Proportion de patients avec des spermatozoïdes mobiles

$\begin{array}{lllllll} & \text { J0 } & \text { J1 } & \text { J2 } & \text { J3 } & \text { J4 } & \text { J5 } \\ \text { Zhu et al, 1997 }(\mathrm{n}=7)[45] & 0 / 7 & & \mathbf{5 / 7} & \mathbf{5 / 7} & & \\ \text { Urman et al, 1998 (n=66) [40] } & 66 / 66 & & 65 / 66 & & \end{array}$

Pourcentage moyen de spermatozoïdes mobiles

Liu et al, $1997(n=9)$ [19]

0

0

0

0

0

0

Angelopoulos et al, 1999

$(\mathrm{n}=3)(\mathrm{a})[2]$

2

8

8

0

0

Angelopoulos et al, 1999

$(\mathrm{n}=1)$ [2]

0

22

20

0

0

Angelopoulos et al, 1999

$(\mathrm{n}=1)$ [2]

0

0

0

0

0

Balaban et al, $1999 \quad(n=70)$ [3]

$\mathbf{0}+33$

Balaban et al, $1999 \quad(n=73)$

+ FSH [3]

$8+71$

J0 à Jn : nombre de jours de culture

Les caractères en gras indiquent la mobilité progressive

(a) : seuls sont pris en compte les échantillons présentant des spermatozoïdes mobiles lors de la ponction

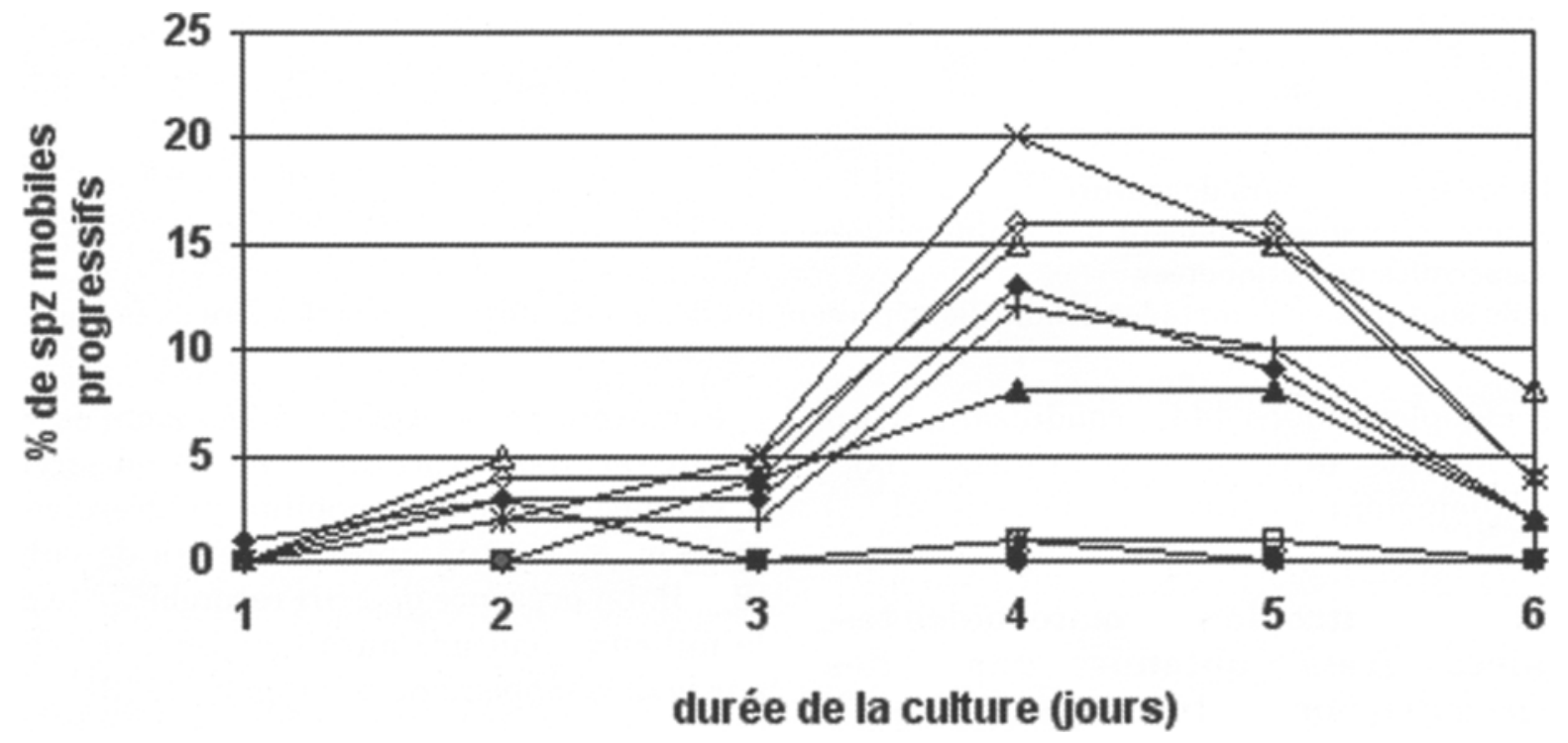

Figure 1 : Evolution de la mobilité progressive (\%) des spermatozoïdes testiculaires au cours de la culture in vitro: Etude dans 11 cas d'azoospermie non obstructive [19]. On note une augmentation appréciable de la mobilité progressive dans 6 cas sur 11. On ne distingue que 8 courbes, les 3 autres sont confondues avec l'axe des abscisses. 
orants vitaux [18, 19]. Par contre d'autres auteurs observent des spermatozoïdes mobiles après 1 à 3 jours de culture [2, 44].

En améliorant leur mobilité, la culture in vitro des spermatozoïdes testiculaires permet de réaliser l'ICSI dans de meilleures conditions. En effet, le temps de recherche de spermatozoïdes mobiles dans ces prélèvements est diminué ( 5 minutes versus 15 minutes par ovocyte) [13] et la proportion d'ovocytes injectés avec des spermatozoïdes mobiles est augmentée [3]. Dans les azoospermies non obstructives, la présence ou non de spermatozoïdes le jour de la ponction testiculaire est difficilement prévisible et l'effet de la culture in vitro en terme de mobilité s'avère aléatoire. Dans ces conditions, il est conseillé de réaliser la ponction testiculaire le jour de la ponction ovocytaire ou éventuellement, d'effectuer une congélation des spermatozoïdes.

\section{Culture in vitro des échantillons testic- ulaires couplée à une cryoconservation (Tableaux 4 et 5 ).}

Dans les azoospermies non obstructives, il est difficile de récupérer suffisamment de spermatozoïdes pour évaluer correctement les effets de la cryoconservation. Aussi, les études sont le plus souvent réalisées à partir d'échantillons provenant de sujets ayant une azoospermie obstructive.

Différents protocoles ont été envisagés pour déterminer les conditions les plus adaptées pour cryoconserver les spermatozoïdes testiculaires. La congélation donne de meilleurs résultats en terme de mobilité et de vitalité des spermatozoïdes, lorsque les tissus sont conservés après dilacération, c'est à dire sous forme d'une suspension hétérogène de cellules testiculaires, plutôt qu'en pièce de biopsie intacte [4].

Deux protocoles ont été testés : congélation/décongélation puis culture ou culture puis congélation/décongélation.

Après décongélation, les résultats apparaissent contradictoires, la culture s'avère délétère [10] ou au contraire bénéfique puisque la mobilité progressive est augmentée [8, 19] (Tableau 4), mais la mobilité totale reste cependant plus faible que celle observée après culture des spermatozoïdes non congelés (Tableau 2).

Les spermatozoïdes testiculaires maintenus en culture pendant 3 jours présentent une meilleure résistance à la congélation/décongélation que les spermatozoïdes non cultivés : $13 \%$ versus $2 \%$ de mobilité [9] ou $17 \%$ versus $4 \%$ [10] (Tableau 5). Cette amélioration est plus importante si la culture a été réalisée à $32^{\circ} \mathrm{C}$ plutôt qu'à $37^{\circ} \mathrm{C}$. Par contre, une nouvelle culture de 24 heures après la décongélation n'entraîne aucune amélioration supplémentaire [41].

Liu et al. [20, 21], ont développé une méthode de cryoconservation des spermatozoïdes mobiles, dans des zones pellucides vides de souris. Cette méthode est particulièrement adaptée pour de faibles quantités de spermatozoïdes. Dans ces conditions, la résistance à la congélation des spermatozoïdes préalablement cultivés pendant 3 jours est identique à celle des spermatozoïdes congelés le jour de la ponction testiculaire, en terme de mobilité (56\% versus. $58 \%$ respectivement) et de vitalité (77\% versus. $74 \%$ respectivement) [21]. Cette méthode reste expérimentale en raison de l'utilisation de matériel animal dont la sécurité sanitaire ne peut pas être garantie, mais elle pourrait être adaptée à des zones pellucides humaines, puisque des congélations de spermatozoïdes, réalisés selon cette technique, ont permis d'obtenir des naissances [42].

4. Taux de fécondation et taux de grossesse après ICSI réalisées avec des spermatozoïdes cultivés in vitro (Tableau $6)$.

La culture des spermatozoïdes est récente et peu d'équipes ont réalisé des ICSI avec de tels spermatozoïdes. La durée de culture est courte ( 1 à 2 jours) et correspond en général au délai entre l'injection d'hCG et la ponction ovocytaire. La réalisation de la ponction testiculaire préalablement à la ponction ovocytaire offre cependant des avantages : une augmentation éventuelle de mobilité des spermatozoïdes permettant ainsi de réaliser l'ICSI dans de meilleures conditions et surtout la suppression éventuelle d'une ponction ovocytaire inutile en 
Tableau 4: Mobilité des spermatozoüdes testiculaires congelés/décongelés et mis en culture in vitro.

\section{Pourcentage moyen de spermatozoïdes mobiles}

\begin{tabular}{|c|c|c|c|c|c|c|c|}
\hline & J0 & $\mathrm{J} 1$ & $\mathrm{~J} 2$ & J3 & $\mathrm{J} 4$ & J5 & J7 \\
\hline Edirisinghe et al, $1996(n=1)[8]$ & $\mathbf{0}$ & 4 & 9 & 8 & 5 & $\mathbf{3}$ & $\mathbf{0}$ \\
\hline Liu et al, $1997(n=15)[19]$ & $2+31$ & & & & & & \\
\hline Emiliani et al, $2000(n=4)[10]$ & 7 & 1 & & & & & \\
\hline
\end{tabular}

J0 à Jn : nombre de jours de culture

Les mobilités notées sont celles observées après décongélation (J0) puis après $\mathrm{x}$ jours de culture in vitro (Jx) dans des azoospermies obstructives

Les caractères en gras indiquent la mobilité progressive

Tableau 5 : Mobilité des spermatozoüdes testiculaires mis en culture in vitro puis congelés/ décongelés.

Pourcentage moyen de spermatozoïdes mobiles

$\begin{array}{llll}\text { J0 } & \text { J1 } & \text { J2 } & \text { J3 }\end{array}$

Van den Bergh et al, 1998**

$(\mathrm{n}=7)[41]$

Emiliani et al, 1999* $(\mathrm{n}=6)$ [9] 2213

Liu et al, $2000^{* * *}(\mathrm{n}=9)[21] \quad 58 \quad 56$

Emiliani et al, $2000^{*}(\mathrm{n}=8)[10] \quad 4 \quad 17$

J0 à Jn : nombre de jours de culture

Les mobilités sont celles observées le jour de la décongélation

*: azoospermies obstructives

**: 5 azoospermies obstructives et 2 azoospermies non obstructives

*** : 6 azoospermies obstructives et 3 azoospermies non obstructives.

Congélation de spermatozoïdes mobiles dans des zones pellucides vides de souris.

Tableau 6 : Taux de fécondation et taux de grossesse après ICSI réalisée en utilisant des spermatozoüdes testiculaires cultivés in vitro.

\begin{tabular}{|c|c|c|c|c|c|}
\hline & \multirow{2}{*}{$\begin{array}{l}\text { Durée de } \\
\text { culture } \\
\text { (jours) }\end{array}$} & \multicolumn{2}{|c|}{$\begin{array}{l}\text { TAUX DE } \\
\text { fécondation }\end{array}$} & \multicolumn{2}{|c|}{$\begin{array}{c}\text { TAUX DE GROSSESSE/ } \\
\text { TRANSFERT }\end{array}$} \\
\hline & & $\begin{array}{l}\text { Azoo. } \\
\text { obst. }\end{array}$ & $\begin{array}{l}\text { Azoo. } \\
\text { non obst. }\end{array}$ & $\begin{array}{c}\text { Azoo. } \\
\text { obst }\end{array}$ & $\begin{array}{l}\text { Azoo. non } \\
\text { obst. }\end{array}$ \\
\hline Nijs et al, $1997^{*}(n=35)[28]$ & 1 & $74^{*}$ & & $31^{*}$ & \\
\hline Urman et al, $1998^{* *}(\mathrm{n}=87)[40]$ & 1,5 & $53^{* *}$ & & $41^{* *}$ & \\
\hline Balaban et al, 1999 sans FSH $(n=70)$ [3] & 1 & & 42,1 & & 30 \\
\hline Balaban et al, $1999+$ FSH $(n=73)[3]$ & 1 & & 68,8 & & 47,8 \\
\hline Hu et al, $1999(n=11)[13]$ & 1 & 60 & & 55 & \\
\hline Hu et al, $1999(n=9)[13]$ & 1 & & 55 & & 33 \\
\hline Jaroudi et al, $1999(n=39)[15]$ & 2 & & 59,6 & & 32,4 \\
\hline
\end{tabular}

Azoo. obst. : azoospermies obstructives Azoo. non obst. : azoospermies non obstructives

* : Azoospermies non étiquetées

** : Des spermatozoïdes épididymaires ont été utilisés dans 11 cas 
cas d'absence de spermatozoïdes. Ainsi, l'injection d'hCG a pu être évitée dans $5 \%$ [15] ou $35 \%$ des cas [40].

La plupart des études portent sur des prélèvements testiculaires mis en culture immédiatement après leur obtention. La culture in vitro des spermatozoïdes testiculaires améliore les taux de fécondation et les taux de grossesse qui sont alors comparables à ceux retrouvés avec des spermatozoïdes éjaculés [13, 40] et plus élevés que ceux obtenus avec des spermatozoïdes testiculaires non cultivés : $60 \%$ [15] versus. $47 \%$ [11]. Cependant, seuls Urman et al. [40] comparent, dans la même étude, les taux de grossesse en ICSI obtenus avec des spermatozoïdes éjaculés non cultivés et des spermatozoïdes testiculaires et/ou épididymaires cultivés. Dans ces deux conditions ils n'observent pas de différence significative $(44,9 \%$ versus. $41,2 \%$ )

D'autre part, à notre connaissance, une seule équipe a utilisé une congélation de spermatozoïdes couplée à une courte incubation (6 heures) et a comparé l'efficacité des spermatozoïdes incubés puis congelés, à celle des spermatozoïdes congelés puis incubés. Dans les deux situations les résultats sont très satisfaisants mais le taux de fécondation semblerait plus élevé lorsque l'incubation est secondaire à la congélation/décongélation : $63,5 \%$ versus. $71,6 \%$ [30].

\section{CONCLUSION}

Le but de la culture in vitro des spermatozoïdes testiculaires est d'essayer de pallier au manque de mobilité de ces spermatozoïdes en les mettant dans des conditions leur permettant de l'acquérir. Les résultats de l'ICSI avec des spermatozoïdes testiculaires devraient ainsi s'en trouver améliorés.

La revue de la littérature montre que l'efficacité de la culture in vitro des spermatozoïdes testiculaires dépend de la nature de l'azoospermie.

Dans les azoospermies obstructives, la culture des spermatozoïdes testiculaires frais permet d'augmenter le pourcentage moyen de spermatozoïdes mobiles. Mais le plus souvent, dès le jour du prélèvement, la mobilité est déjà cor- recte, la culture perd alors de son intérêt, sauf si une maturation devait exister.

Dans les azoospermies non obstructives, lorsque des spermatozoïdes sont retrouvés dans la ponction testiculaire, ce qui n'est pas toujours le cas (jusqu'à $40 \%$ de ponctions négatives. [32]), leur mobilité est toujours faible : la culture in vitro paraît donc justifiée. De plus, le prélèvement testiculaire réalisé préalablement à la ponction ovocytaire permet d'éviter l'injection d'hCG lorsqu'aucun spermatozoïde n'est retrouvé. Les résultats restent cependant aléatoires puisqu'il n'est pas possible de prévoir si la culture s'accompagnera d'un gain de mobilité ou au contraire d'une perte de vitalité, les deux situations ayant été observées.

La nature des milieux de culture semble être un facteur important. Généralement, il s'agit de milieux chimiquement définis, classiquement utilisés dans les cultures cellulaires. Toutefois, certaines améliorations peuvent être envisagées et parmi les facteurs susceptibles d'avoir un effet bénéfique sur la mobilité, nous retiendrons la FSH recombinante.

Les meilleurs résultats en terme d'acquisition de mobilité sont observés après trois à quatre jours de culture, mais avec de tels délais, il est difficile de synchroniser la ponction testiculaire et la ponction ovocytaire. La cryoconservation pallie en partie à cet inconvénient, évite une ponction ovocytaire inutile, et peut être associée à une culture in vitro. Cette dernière semble plus efficace si elle est réalisée avant plutôt qu'après l'étape de congélation/décongélation.

Finalement, bien que les résultats soient encore fragmentaires, il semble que la meilleure solution serait de réaliser la culture immédiatement après la biopsie testiculaire, de congeler les spermatozoïdes, puis de les décongeler le jour de la tentative d'ICSI.

La culture in vitro des spermatozoïdes testiculaires peut constituer une voie de recherche à développer pour améliorer les résultats de l'ICSI et constitue un bon modèle d'étude des mécanismes, encore très peu connus, d'acquisition de la mobilité des spermatozoïdes. 


\section{RÉFÉRENCES}

1. AITKEN R.J. : Possible redox regulation of sperm motility activation. J. Androl., 2000, 21 : 491-496.

2. ANGELOPOULOS T., ADLER A., KREY L., LICCIARDI F., NOYES N., MC CULLOUGH A. : Enhancement or initiation of testicular sperm motility by in vitro culture of testicular tissue. Fertil. Steril., 1999, 71: 240-243.

3. BALABAN B., URMAN B., SERTAC A., et al. : In vitro culture of spermatozoa induces motility and increases implantation and pregnancy rates after testicular sperm extraction and intracytoplasmic sperm injection. Hum. Reprod., 1999, 14 : 2808-2811.

4. CRABBE E., VERHEYEN G., TOURNAYE H., VAN STEIRTEGHEM. : Freezing of testicular tissue as a minced suspension preserves sperm quality better than whole-biopsy freezing when glycerol is used as a cryprotectant. Int. J. Androl., 1999, 22 : 43-48.

5. CRAFT I., TSIRIGOTIS M., ZHU J.J. : In vitro culture of testicular sperm. Lancet, 1995, $25: 1438$.

6. DE CROO I., VAN DER ELST J., EVERAERT K., DE SUTTER P., DHONT M. : Fertilization, pregnancy and embryo implantation rates after ICSI with fresh or frozen testicular spermatozoa. Hum. Reprod., 1998, $13: 1893-1897$.

7. DEVROEY P., LIU J., NAGY Z., et al. : Pregnancies after testicular sperm extraction and intracytoplasmic sperm injection in non obstructive azoospermia. Hum. Reprod., 1995, $10:$ 1457-1460.

8. EDIRISINGHE W.R., JUNK S.M., MATSON P.L., YOVITCH J.L. : Changes in motility patterns during in vitro culture of fresh and frozen/thawed testicular and epididymal spermatozoa : implications for planning treatment by intracytoplasmic sperm injection. Hum. Reprod., 1996, 11 : 2474-2476.

9. EMILIANI S., VAN DEN BERGH M., BIRAMANE J., ENGLERT Y. : Improved motility after in vitro culture of testicular spermatozoa in obstructive azoospermia results in better post thaw survival: a benefit for the patient and the laboratory. Hum. Reprod., 1999, Abstract Book of the $15^{\text {th }}$ annual meeting of the ESHRE : O226.

10. EMILIANI S., VAN DEN BERGH M., VANNIN A.S., BIRAMANE J., VERDOOT M., ENGLERT Y. : Increased sperm motility after in vitro culture of testicular biopsies from obstructive azoospermic patients results in better post-thaw recovery rate. Hum. Reprod., 2000, 15 : 2371-2374.

11. FIVNAT : Bilan de l'année 1998. Dossier FIVNAT 99, 1999.

12. HABERMANN H., SEO R., CIESLAK J., NIEDERBERGER C., PRINS G.S., ROSS L. : In vitro outcomes after intracytoplasmic sperm injection with fresh or frozen-thawed testicular spermatozoa. Fertil. Steril., 2000, $73:$ 955-960.
13. HU Y., MAXSON W.S., HOFFMAN D.I., ORY S.J., LICHT M.R., EAGER S. : Clinical application of intracytoplasmic sperm injection using in vitro cultured testicular spermatozoa obtained the day before egg retrieval. Fertil. Steril., 1999, 72 : 666-669.

14. HUANG F.J., CHANG S.Y., TSAI M.Y., et al. : Clinical implications of intracytoplasmic sperm injection using cryopreserved testicular spermatozoa from men with azoospermia. J. Reprod. Med., 2000, 45 : 310316.

15. JAROUDI K., COSKUN S., HOLLANDERS J., et al.: Advanced surgical sperm recovery is a viable option for intracytoplasmic sperm injection in patients with obstructive or non obstructive azoospermia. Fertil. Steril., 1999, 72 : 479-483.

16. KAHRAMAN S., TASDEMIR M., TASDEMIR I., et al.: Pregnancies achieved with testicular and ejaculated spermatozoa in combination with intracytoplasmic sperm injection in men with totally or initially immotile spermatozoa in the ejaculate. Hum. Reprod., 1996, 11 : 1343-1346.

17. LIU J., NAGY Z., JORIS H., et al. : Analysis of 76 total fertilization failure cycles out of 2732 intracytoplasmic sperm injections cycles. Hum. Reprod., 1995, $10: 2630-2636$.

18. LIU J., GARCIA J.E., BARAMKI T.A. : The difference in outcome of in vitro culture of human testicular spermatozoa between obstructive and non obstructive azoospermia. Hum. Reprod., 1996, 11 : 1587-1588.

19. LIU J., TSAI Y.L., KATZ E., COMPTON G., GARCIA J.E., BARAMKI T.A. : Outcome of in vitro culture of fresh and frozen thawed human testicular spermatozoa. Hum. Reprod., 1997, 12 : 1667-1672.

20. LIU J., ZHENG X.Z., BARAMKI T.A., YAZIGI R.A., COMPTON G., KATZ E. : Comparison of viability between fresh human testicular spermatozoa that were cryopreserved after in vitro culture for 3 days. Fertil. Steril., 1999, 72, suppl.1 : S99.

21. LIU J., ZHENG X.Z., BARAMKI T.A., COMPTON G., YAZIGI R.A., KATZ E. : Cryopreserved of a small number of fresh human testicular spermatozoa and testicular spermatozoa cultured in vitro for 3 days in an empty zona pellucida. J. Androl., 2000, 21 : 409413.

22. MOORE H.D.M., CURRY M.R., PENFOLD L.M., PRYOR J.P. : The culture of human epididymal epithelium and in vitro maturation of epididymal spermatozoa. Fertil. Steril., 1992, 58 : 776-783.

23. MULHALL J.P., CUNNINGHAM D., BURGESS C. : The prevalence of mature whole spermatozoa in the testicular parenchyma of males with non obstructive azoospermia. J. Urol., 1996, $155:$ 365A.

24. NAGY Z.P., LIU J., JORIS H., et al. : The result of intracytoplasmic sperm injection is not related to any of the three basic sperm parameters. Hum. Reprod., 1995 a, $10: 1123-1129$. 
25. NAGY Z., SILBER S., LIU J., DEVROEY P., JANSSENWILLEN C., VAN STEIRTEGHEM A.C. : Using ejaculated, fresh, and frozen-thawed epididymal and testicular spermatozoa gives rise to comparable results after intracytoplasmic sperm injection. Fertil. Steril., 1995 b, 63 : 808-815.

26. NAGY Z., JORIS H., VERHEYEN G., TOURNAYE H., DEVROEY P., VAN STEIRTEGHEM A.C. : Correlation between motility of testicular spermatozoa, histology and the outcome of intracytoplasmic sperm injection. Hum. Reprod., 1998, 13 : 890-895.

27. NIJS M., VANDERZWALMEN P., VANDAMME B., et al. : Fertilizing ability of immotile spermatozoa after intracytoplasmic sperm injection. Hum. Reprod., $1996,11: 2180-2185$.

28. NIJS M., SEGAL-BERTIN B., VANDAMME P., VANDERZWALMEN P., LEJEUNE B., SCHOYSMAN R. : In vitro culture of testicular spermatozoa : an improvement for the ICSI outcome? J. Assist. Reprod. Genet., 1997, 14, suppl. : 75S.

29. NIJS M., BERTIN G., VANDAMME B., VANDERZWALMEN P., LEJEUNE B., SCHOYSMAN R. : In vitro culture of testicular and epididymal spermatozoa : immotility is non viability? Andrology in the nineties, 1998, Book of abstracts and miniposters : 39 .

30. NUNEZ-CALONGE R., CORTES S., AUGUSTIN S., GONZALES CASBAS J.M., HERNANDEZ E., CABARELLO P. : Effect of frozen-thawed human testicular spermatozoa incubation on ICSI outcome. Hum. Reprod., 2000, Abstract Book 1 of the 16th annual meeting of the ESHRE : P109.

31. PALERMO G.D., JORIS H., DEVROEY P., VAN STEIRTEGHEM A.C. : Pregnancies after intracytoplasmic injection of single spermatozoon into an oocyte. Lancet, 1992, $340: 17-18$.

32. PALERMO G.D., SCHLEGEL P.N., HARIPRASHAD J.J., et al. : Fertilization and pregnancy outcome with intracytoplasmic sperm injection for azoospermic men. Hum. Reprod., 1999, 14: 741-748.

33. PRINS G.S., DOLGINA R., STUDNEY P., KAPLAN B., ROSS L., NIEDERBERGER C. : Quality of cryopreserved testicular sperm in patients with obstructive and non obstructive azoospermia. J. Urol., 1999, 161 : 1504-1508.

34. RIVES N., SIBERT L., CLAVIER B., DELABTROYE V., MARPEAU L., MACE B. : Full term delivery following intracytoplasmic sperm injection with frozenthawed immotile testicular spermatozoa. Hum. Reprod., 1998, $13:$ :3399-3401.

35. SHIBAHARA H., HAMADA Y., HASEGAWA A., et al.:
Correlation between the motility of frozen-thawed epididymal spermatozoa and the outcome of intracytoplasmic sperm injection. Int. J. Androl., 1999, 22 : 324-328.

36. SHULMAN A., FELDMAN B., MADGAR I., LEVRON J., MASHIACH S., DOR J. : In vitro fertilization treatment for severe mal factor: the fertilization potential of immotile spermatozoa obtained by testicular extraction. Hum. Reprod., 1999, 14 : 749-752.

37. SILBER S.J., NAGY Z.P., LIU J., GODOY H., DEVROEY P., VAN STEIRTEGHEM A.C. : Conventional in vitro fertilization versus intracytoplasmic sperm injection for patients requiring microsurgical sperm aspiration. Hum. Reprod., 1994, 9 : 1705-1709.

38. SILBER S.J., VAN STEIRTEGHEM A.C., LIU J., NAGY Z., TOURNAYE H., DEVROEY P. : High fertilization and pregnancy rate after intracytoplasmic sperm injection with spermatozoa obtained from testicle biopsy. Hum. Reprod., 1995, $10: 148-152$.

39. UBALDI F., NAGY Z.P., RIENZI L., et al. : Reproductive capacity of spermatozoa from men with testicular failure. Hum. Reprod., 1999, 14 : 27962800.

40. URMAN B., ALATAS C., AKSOY S., NUHOGLU A., SERTAC A., BALABAN B. : Performing testicular or epididymal sperm retrieval prior to the injection of hCG. J. Assist. Reprod. Genet., 1998, 15 : 125-128.

41. VAN DEN BERGH M., EMILIANI S., BIRAMANE J., VANNIN A.S., ENGLERT Y. : Prolonged culture at $32^{\circ} \mathrm{C}$ and $37^{\circ} \mathrm{C}$ of fresh and frozen testicular biopsies. Hum. Reprod., 1998, Abstract Book 1 of the 14th annual meeting of the ESHRE : O219.

42. WALMSLEY R., COHEN J., FERRARA-CONGEDO T., REING A., GARRISI J. : The first births and ongoing pregnancies associated with sperm cryopreservation within evacuated egg zonae. Hum. Reprod., 1998, 13, suppl $4: 61-70$.

43. WHO : WHO laboratory manual for the examination of human semen and sperm-cervical mucus interaction. $3^{\text {rd }}$ edn, Cambridge University Press, Cambridge, UK, 1992.

44. ZHU J., TSIRIGOTIS M., PELEKANOS M., CRAFT I. : In vitro maturation of human testicular spermatozoa. Letter, Hum. Reprod., 1996, 11 : 231-232.

45. ZHU J., MENIRU G.I., CRAFT I. : In vitro maturation of human testicular sperm in patients with azoospermia. J. Assist. Reprod. Genet., 1997, 14 : 361363. 


\begin{abstract}
Testicular spermatozoa for ICSI: benefits of in vitro culture. A review.

B. SCHUBERT, G. GRIZARD, D. BOUCHER
\end{abstract}

The number of ICSI cycles performed with testicular spermatozoa has increased dramatically over recent years. However, one of the technical limitations of this approach concerns the extremely reduced motility of testicular spermatozoa. However, increased sperm motility was observed after incubating testicular samples for several hours. Therefore, in order to improve ICSI success rates, several authors have tested the effect of previous in vitro culture. We present a review of the literature on this subject.

In vitro culture does not appear to be very useful in cases of obstructive azoospermia, as, apart from possible sperm "maturation" during this culture phase, a high proportion of motile spermatozoa is usually already observed prior to in vitro culture.

The benefits of in vitro culture appear to be greater in the case of non-obstructive azoospermia, as when spermatozoa are present on the biopsy, they are usually immobile. However, discordant results have been published: after in vitro culture, spermatozoa have been reported to be either motile or mostly dead.
Regardless of the type of azoospermia, the best results are obtained after 3-4 days of in vitro culture. Addition of recombinant FSH to the culture medium also appears to be effective.

Cryopreservation of testicular biopsies may also be associated with in vitro culture and the in vitro culture/freezing sequence appears to give better results than the freezing/in vitro culture sequence.

Very few studies have reported the results of ICSI using frozen in vitro cultured spermatozoa, as most published studies concern fresh spermatozoa, used after 1-2 days of in vitro culture with satisfactory fertilization and pregnancy rates.

In vitro culture of testicular spermatozoa may therefore constitute an interesting research approach to improve the results of ICSI when the number of spermatozoa and/or motility are very low. In addition, in vitro culture of testicular spermatozoa appears to be a good tool to study the mechanisms of acquisition of motility, which are still poorly understood.

Key words: testicular spermatozoa, in vitro culture, azoospermia, freezing/thawing 